\title{
Enseñanza aprendizaje del diseño arquitectónico y la educación para el desarrollo sostenible en universidades de Huancayo
}

\author{
Teaching-learning in architectural design and education for \\ sustainable development in the universities of Huancayo
}

\author{
Adolfo Gustavo Concha Flores ${ }^{1}$ \\ Universidad Nacional del Centro del Perú \\ gusstock@hotmail.es
}

\section{RESUMEN}

El objetivo fue describir las variables, enseñanza aprendizaje del diseño arquitectónico y la Educación para el Desarrollo Sostenible en las universidades de Huancayo, luego establecer sus correlaciones. Este trabajo de investigación fue de nivel descriptivo correlacional, diseño no experimental, transversal. El instrumento de investigación fue la ficha de análisis de contenido, que para su validez se aplicó el Índice Kappa de Fleiss, que determinó un valor para ambas variables de 0,93 (acuerdo casi perfecto). La prueba de confiabilidad utilizó el Coeficiente Alfa de Cronbach cuya medida para ambas variables fue de 0,81 (Muy alto). La muestra utilizada fue no probabilístico, a juicio del investigador. Entre los resultados se pueden mencionar: la variable Enseñanza aprendizaje del diseño arquitectónico y la variable Fundamentación epistemológica de la EDS tienen puntuaciones de distribución de las medianas, con valores de $M e=0,155$ para la $U N C P, M e=0,14$ para la UPLA y $M e=0,1375$ para la UC, describiendo que existe acuerdos bajos entre estas. También se concretizaron un intervalo de desviación estándar con valores de 0,27 a -0,035 que determinaron una alta separación y la tendencia a bajos acuerdos entre las variables. Entre las conclusiones tenemos que existen acuerdos bajos entre las variables y de conformidad al coeficiente de correlación, la enseñanza de la arquitectura mantiene correlaciones mínimas, que permitió mostrar que se encuentra al margen del valor y la significancia que posee la Educación para el Desarrollo Sostenible.

Palabras clave: Enseñanza aprendizaje, diseño arquitectónico, educación para el desarrollo Sostenible.

\begin{abstract}
The aim of the study was to describe the variables teaching and learning, and education of architectural design for sustainable development in Huancayo Universities, also to establish their correlation relationships. This research was of correlational and descriptive level, not experimental, and had a cross-sectional design. The content analysis card was the instrument, it applied the Fleiss Kappa index to determine a value for both variables of 0,93 (almost perfect agreement). The Reliability test used the Cronbach's alpha coefficient whose measure for both variables was 0,81 (very high). The sampling used was non probabilistic, namely by criterion. As results, both learning and teaching of architectural design and the SDE epistemological foundation variables had median distribution scores: UNCP Me =0,155, UPLA Me =0,14 and UC Me $=0,1375$, showing that there are low agreements among these. In addition, the standard deviation ranged from 0,27 to 0,035 values that determined high dispersion and a tendency to low agreements between variables. To conclude, in accordance with the correlation coefficient there are low agreements between variables; and the teaching of architecture has minimal correlations, showing that regardless of the importance of Education for Sustainability, appropriate steps have yet to be taken.
\end{abstract}

Keywords: Teaching and learning, architectural design, education for sustainable development.

Historial del artículo:

Recibido: 8 de marzo de 2015. Aprobado: 6 de mayo 2015. Disponible en línea: 30 de junio de 2015

1 Dr. en Ciencias de la Educación, Dr. en Ciencias Ambientales y Desarrollo Sostenible, docente de la Facultad de Arquitectura de la

Universidad Nacional del Centro del Perú. 


\section{INTRODUCCIÓN}

El trabajo de investigación propone la viabilidad de una estrategia pertinente al campo de la Educación para el Desarrollo Sostenible (EDS), pero, desde la óptica de la enseñanza aprendizaje del diseño arquitectónico en las universidades de Huancayo que poseen facultades de arquitectura, que se muestran como columna vertebral de todo el conjunto de procesos pedagógicos, que surgen como las alternativas que posibiliten la articulación equilibrada entre el medio físico natural y el medio cultural, y que a su vez incorpore en el nivel de la educación superior el paradigma del desarrollo sostenible, como vector direccional de la preservación del planeta, debido a que los problemas ambientales que alberga y que están aconteciendo, se deben sobre todo al esquema actual de desarrollo de la sociedad y que no tiene precedentes históricos. Uno de esos problemas lo constituye la correlación dinámica entre el crecimiento demográfico, y que en el momento actual se muestra incontrolable, frente a una casi carencia en el uso de las energías renovables, y que han sido una de las principales causas de su degradación y deterioro.

En base a las consideraciones expuestas se formuló el problema general de investigación siguiente: ¿̇qué relación existe entre la enseñanza aprendizaje del diseño arquitectónico y la Educación para el Desarrollo Sostenible en las universidades de Huancayo? Mientras el objetivo general fue: determinar el nivel de relación que existe entre la enseñanza aprendizaje del diseño arquitectónico y la Educación para el Desarrollo Sostenible en las universidades de Huancayo; y se planteó la siguiente hipótesis general: existe una relación mínima entre la enseñanza aprendizaje del diseño arquitectónico y la Educación para el Desarrollo Sostenible en las universidades de Huancayo.

Dentro de este contexto la enseñanza aprendizaje de la disciplina de la arquitectura, cuyo sinónimo de replica se encuentra en el diseño arquitectónico en sus diferentes variantes de nombres, muestra serias carencias para poder enfrentar la crisis ambiental que se vive, debido a que aún prevalecen modelos, planes y diseños curriculares con una postura académica tradicional que privilegia las racionalidades socio económicas, soslayando la dimensión ambiental que es inherente al desarrollo sostenible, es decir, no articula adecuadamente la enseñanza aprendizaje del diseño arquitectónico y la Educación para el Desarrollo Sostenible en el ámbito de las universidades locales de la metrópoli de Huancayo.

En la coyuntura mundial actual, uno de los puntos de referencia relevantes en la discusión internacional sobre la educación, especialmente en el nivel superior, es la de establecer el paradigma del desarrollo sostenible, es por ese motivo que las Naciones Unidas mediante la UNESCO, ha determinado que entre los años del 2005 al 2014 sea llamada la Década de la Educación para el Desarrollo Sostenible (1). La EDS enfatiza la necesidad de una educación con objeto de alcanzar los distintos aspectos necesarios para resolver los requerimientos de la sostenibilidad, indica que en las escuelas se deben proporcionar habilidades de aprendizaje para conocer, para vivir en compañía, para hacer y para ser (2). Además, considera la reorientación de los programas educativos existentes (3). Es decir, el currículo actual, desde la educación para lactantes hasta la educación superior, debe ser revisado en el contexto de la sustentabilidad.

Desde la perspectiva de la enseñanza aprendizaje del diseño arquitectónico, también es muy poco lo que se ha hecho sobre este tema, porque sus objetivos y metas son complejas, debido a que se pretende preparar a todas las personas, involucradas en el quehacer del diseño arquitectónico independientemente de su nivel académico y de su condición social, en diseñar, planificar, enfrentar y resolver las amenazas que pesan sobre nuestro planeta (1), y en la coyuntura actual, se pudo observar en las universidades estudiadas, que existe una notoria carencia de modelos, planes y diseños curriculares que se encuentren influenciados por la EDS, y que son instrumentos de gestión importantes, que darán la partida de nacimiento a esta novedosa pedagogía, siendo este el motivo por el que se realiza este estudio, y que se enmarca dentro de la línea de investigación de la Educación para el Desarrollo Sostenible.

\section{MATERIAL Y MÉTODOS}

El tipo de investigación fue una investigación sustantiva (4), debido a que contiene dos finalidades de estudio: El primero, inherente al estudio de investigación descriptiva y que actuó como condicionante, y cuyo propósito fue describir, analizar e interpretar acuerdos o no acuerdos que existen entre las variables $V x=$ (Enseñanza aprendizaje del diseño arquitectónico) y $V_{y}=$ (Educación para el Desarrollo Sostenible). El segundo, fue un trabajo de investigación correlacional, que se manifestó como determinante y que actuó en la correlación que existe o no existe entre las variables $V x$ y $V y$, con la finalidad de establecer relativamente argumentos explicativos y predictivos.

El alcance de la investigación fue descriptivo (5), debido a que el objetivo general permitió describir acuerdos que existen entre las variables, $V x$ y $V y$, y el correlacional que de concordancia al objetivo general, analizo la relación que existe entre las variables: $V x$ y la Vy.

El método de investigación fue de análisis de contenido (6), que significo el análisis del contenido del diseño 
curricular, plan de estudios, sílabos y entrevista a docentes.

El diseño de investigación fue mixto. Para la investigación descriptiva se uso el diseño siguiente:

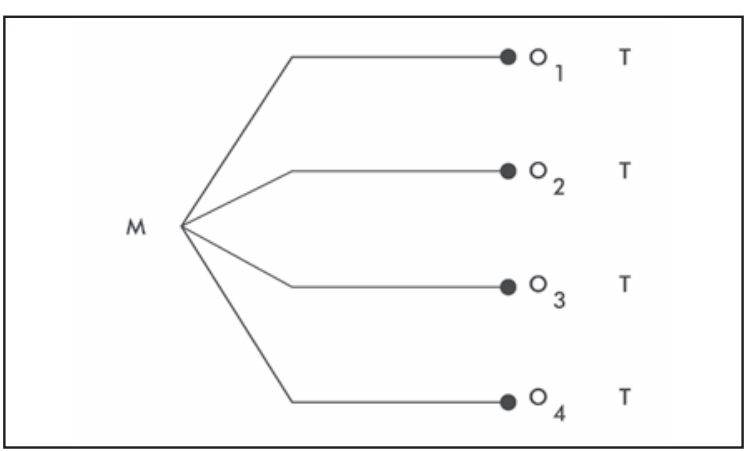

Dónde:

$(M)=$ Represento a la muestra seleccionada que identifico a cada una de las Facultades de Arquitectura de las universidades.

$(\mathrm{O})=$ Represento las observaciones realizadas al aplicar el instrumento ficha de análisis de contenido. Se utilizó el método siguiente: O1 = (Talleres nivel básico / Fundamento epistemológico de la EDS); O2 = (Talleres nivel intermedio /Teorías de la EDS), O3 = (Talleres nivel avanzado / Modelos del aprendizaje cognitivo de la EDS) y $\mathrm{O} 4=$ (Talleres nivel profesional / Enfoques pedagógicos de la EDS).

$(T)=$ Significó que la aplicación de pruebas fue realizada en el año 2012, Semestres 2012-I y 2012-II.

Y para la investigación correlacional se diseñó el siguiente modelo:

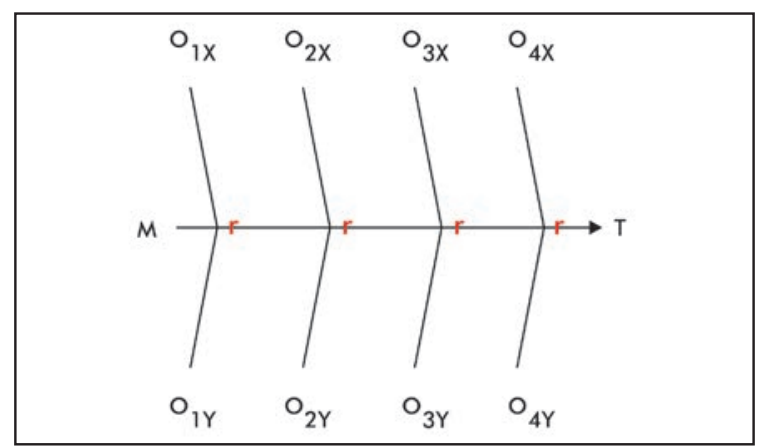

Dónde:

$(M)=$ Represento a cada una de las Facultades de Arquitectura de las universidades.

$(O)=$ Represento las correlaciones que se obtuvieron de las variables al aplicar el instrumento ficha de análisis de contenido en la muestra de estudio.
Se utilizó el procedimiento siguiente: $(\mathrm{O} 1 \mathrm{X}=$ Talleres nivel básico $r \quad 01 Y=$ Fundamento epistemológico de la EDS); (O2X= Talleres nivel intermedio $r \quad \mathrm{O} 2 Y=$ Teorías de la EDS), $(\mathrm{O} 3 \mathrm{X}=$ Talleres nivel avanzado $r \quad \mathrm{O} 3 \mathrm{Y}=$ Modelos del aprendizaje cognitivo de la EDS) y $(\mathrm{O} 4 \mathrm{X}=$ Talleres nivel profesional $r \mathrm{O} 4 \mathrm{Y}=$ Enfoques pedagógicos de la EDS). El objetivo fue de establecer correlaciones de covarianza de las $V x$ y $V y$.

$(r)=$ Represento las correlaciones establecidas, $y$

$(T)=$ Significó que la aplicación de pruebas fue realizada en el año 2012, Semestres 2012-I y 2012-II.

La muestra fue No Probabilística o a juicio del investigador (7), debido a que este tipo de técnica muestral se adecuo al propósito y alcance de la investigación descriptivo-correlacional propuesta.

Las universidades seleccionadas fueron: Universidad Nacional del Centro del Perú (UNCP), Universidad Peruana Los Andes (UPLA) y la Universidad Continental (UC).

El instrumento de recopilación de datos fue la ficha de análisis de contenido, que fue estructurado para registrar la presencia o ausencia de información, sobre las variables: $V x=$ Enseñanza aprendizaje de la arquitectura y $V y=$ Educación para el Desarrollo Sostenible.

La escala fue dicotómica, que acepto dos alternativas (SI-NO). La Prueba de Validez se concretizo con el Índice Kappa de Fleiss para la concordancia tres de expertos concretizando el grado de acuerdo total de 0,93 (Casi perfecto) y con un coeficiente de Confiabilidad del Alfa de Cronbach de 0,75 (Alto), mientras la Confiabilidad determino el grado de acuerdo total de 0,94 (Casi perfecto) y con un coeficiente de Confiabilidad de Alfa de Cronbach de 0,86 (Muy Alto).

\section{RESULTADOS}

La enseñanza aprendizaje de la arquitectura en los niveles básicos y la fundamentación epistemológica de la EDS en las universidades de Huancayo

Los datos de la tabla $N^{\circ} 1$, indican para la variable Vxm (Enseñanza aprendizaje del diseño arquitectónico en los niveles básicos nivel básico I, II y III) y para la Vym (Fundamentación epistemológica de la EDS) puntuaciones de distribución de las medianas, con valores de $M e=0,155$ para la UNCP, UPLA Me $=0,14$ y la $\mathrm{UC} M e=0,1375$; estos datos han permitido describir las incertidumbres que existen y permitió describir que 
existe acuerdos bajos entre estas.

Por otro lado, en la tabla $\mathrm{N}^{\circ} 2$, se observó que el coeficiente de rho de Spearman, para los niveles de diseños básicos de las facultades de arquitectura de la UNCP fue de $r S=0,14$, UPLA $r S=-0,18$ y la UC $r S=0,134$, que sugirió correlaciones mínimas, con direcciones positivas para la UNCP y la UC, manifestando relaciones directamente proporcionales, que afirman la clara inclinación a la ausencia en los modelos curriculares de la Educación para el Desarrollo Sostenible y la fundamentación epistemológica, que produjo deficiencia en la enseñanza del diseño arquitectónico en los niveles básicos; para la UC fue inverso por la dirección negativa que adquirió su correlación. Estos hechos detectaron propuestas de diseños con una clara ausencia de creatividad sostenible, debido a que los docentes muestran en cierto modo una indiferencia a los temas de sostenibilidad o medio ambiente, porque no cuentan con la especialización respectiva.

Como $1,03<1,98$, entonces el estadístico calculado cae en la zona de rechazo, por lo tanto se rechaza la alterna $\mathrm{H}_{1}$ y se acepta la hipótesis nula $\mathrm{H}_{0}$, con lo que se concluye que. "No existe correlación entre la enseñanza aprendizaje del diseño arquitectónico en los niveles básicos y la fundamentación epistemológica de la Educación para el Desarrollo Sostenible en las universidades de Huancayo", comprobándose así la primera hipótesis específica.

\section{La enseñanza aprendizaje del diseño arquitectónico en los niveles intermedios y las teorías de la EDS en las universidades de Huancayo}

Los datos de la tabla $N^{\circ} 3$, indican para la variable Vxm (Enseñanza aprendizaje del diseño arquitectónico en los niveles intermedios) y para la Vym (Teorías de la EDS) puntuaciones en las medianas: para la UNCP $M e=0,185$, UPLA $M e=0,175$, y $\cup C M e=0,145$; estas situaciones detallan los grados de incertidumbre que se tiene entre cada variable y la confirmación de acuerdos bajos para cada facultad de arquitectura en los niveles de estudios especificados.

Tabla № 1: Resumen y diagnóstico a nivel de la mediana de las variables: Enseñanza aprendizaje del diseño arquitectónico en los niveles básicos y la fundamentación epistemológica de la EDS.

\begin{tabular}{lccc}
\hline Variables & $\begin{array}{c}\text { Universidades } \\
\text { con facultades } \\
\text { de arquitectura }\end{array}$ & $\begin{array}{c}\text { Mediana para } \\
\text { datos } \\
\text { agrupados (Me) }\end{array}$ & Diagnóstico \\
\hline $\begin{array}{l}\text { Enel de diseño } \\
\text { básico }\end{array}$ & 0,155 & Acuerdo bajo \\
$\begin{array}{l}\text { del diseño arquitectónico } \\
\text { en los niveles básicos } \\
\text { y la fundamentación } \\
\text { epistemológica de la } \\
\text { EDS. }\end{array}$ & $\begin{array}{c}\text { Nivel de diseño } \\
\text { básico }\end{array}$ & 0,14 & Acuerdo bajo \\
& UC & 0,1375 & Acuerdo bajo \\
\hline
\end{tabular}

Tabla № 2: Resumen y diagnóstico a nivel correlacional de las variables: Enseñanza aprendizaje del diseño arquitectónico en los niveles y la fundamentación epistemológica de la EDS.

\begin{tabular}{|c|c|c|c|}
\hline Variables & $\begin{array}{l}\text { Universidades } \\
\text { con facultades } \\
\text { de arquitectura }\end{array}$ & $\begin{array}{c}\text { Correlación } \\
\qquad\left(r_{s}\right)\end{array}$ & Diagnóstico \\
\hline Enseñanza aprendizaje & $\begin{array}{c}\text { UNCP } \\
\text { Nivel de diseño } \\
\text { básico }\end{array}$ & 0,14 & $\begin{array}{c}\text { Correlación } \\
\text { mínima }\end{array}$ \\
\hline $\begin{array}{l}\text { del diseño arquitectónico } \\
\text { en los niveles básicos } \\
\text { y la fundamentación }\end{array}$ & $\begin{array}{l}\text { UPLA } \\
\text { Nivel de diseño } \\
\text { básico }\end{array}$ & $-0,18$ & $\begin{array}{l}\text { Correlación } \\
\text { mínima } \\
\text { negativa }\end{array}$ \\
\hline $\begin{array}{l}\text { epistemológica de la } \\
\text { EDS. }\end{array}$ & $\begin{array}{l}\text { UC } \\
\text { Nivel de diseño } \\
\text { básico }\end{array}$ & 0,134 & $\begin{array}{c}\text { Correlación } \\
\text { mínima }\end{array}$ \\
\hline
\end{tabular}


Resumen de la prueba de la hipótesis 1: No existe correlación entre la enseñanza aprendizaje del diseño arquitectónico en los niveles básicos y la fundamentación epistemológica de la Educación para el Desarrollo Sostenible en las universidades de Huancayo.

\begin{tabular}{llll}
\hline \multicolumn{1}{c}{$\begin{array}{c}\text { Hipótesis nula } \\
\mathrm{H}_{\mathrm{O}}: \mu=\mu 0\end{array}$} & $\begin{array}{c}\text { Hipótesis alterna } \\
\mathrm{H}_{1}: \mu \neq \mu_{0}\end{array}$ & $\begin{array}{c}\text { Nivel de } \\
\text { significancia }\end{array}$ & $\begin{array}{c}\text { Test } \\
\text { estadístico }\end{array}$ \\
\hline $\begin{array}{l}\text { No Existe correlación entre la } \\
\text { enseñanza aprendizaje del diseño } \\
\text { arquitectónico en los niveles } \\
\text { básicos y la fundamentación } \\
\text { epistemológica de la EDS. }\end{array}$ & $\begin{array}{l}\text { arquitectónico en los niveles } \\
\text { básicos y la fundamentación } \\
\text { epistemológica de la EDS. }\end{array}$ & $5 \%$ & Zc $=1,03$ \\
\hline
\end{tabular}

Seguidamente se detalló en la tabla $N^{\circ} 4$ que los coeficientes de rho de Spearman para los niveles intermedios de las facultades de arquitectura de la UNCP $r S=0,14$, UPLA $r S=-0,10$, y $U C r S=0,13$; determinando para los tres casos correlaciones mínimas, con direcciones positivas para la UNCP y la $U C$, y relaciones directamente proporcionales, que se tradujo en la influencia mínima que tienen las teorías que fundamentan la Educación para el Desarrollo Sostenible en los currículos, lo que ha determinado la ausencia de la buena calidad de la enseñanza del diseño arquitectónico en los niveles intermedios, hecho que es inversamente proporcional en la UPLA; lo que trajo consigo propuestas de diseños arquitectónicos carentes de innovación sostenible.

Como $1,23<1,35$, entonces el estadístico calculado cae en la zona de rechazo, por lo tanto se rechaza la alterna $H_{1}$ y se acepta la hipótesis nula $H_{0}$ con lo que se concluye que "No hay correlación entre la enseñanza aprendizaje del diseño arquitectónico en los niveles intermedios y las teorías que fundamentan

Tabla № 3: Resumen y diagnóstico a nivel de la mediana de las variables: Enseñanza aprendizaje del diseño arquitectónico en los niveles intermedios y las Teorías de la EDS.

\begin{tabular}{|c|c|c|c|}
\hline Variables & $\begin{array}{l}\text { Universidades } \\
\text { con facultades } \\
\text { de arquitectura }\end{array}$ & $\begin{array}{l}\text { Mediana para } \\
\text { datos } \\
\text { agrupados (Me) }\end{array}$ & Diagnóstico \\
\hline \multirow{3}{*}{$\begin{array}{l}\text { Enseñanza } \\
\text { aprendizaje del diseño } \\
\text { arquitectónico en los } \\
\text { niveles intermedios y las } \\
\text { teorías de la EDS. }\end{array}$} & $\begin{array}{c}\text { UNCP } \\
\text { Nivel de diseño } \\
\text { intermedio }\end{array}$ & 0,185 & Acuerdo bajo \\
\hline & $\begin{array}{c}\text { UPLA } \\
\text { Nivel de diseño } \\
\text { intermedio }\end{array}$ & 0,175 & Acuerdo bajo \\
\hline & $\begin{array}{c}\text { UC } \\
\text { Nivel de diseño } \\
\text { intermedio }\end{array}$ & 0,145 & Acuerdo bajo \\
\hline
\end{tabular}

Tabla N ${ }^{\circ}$ 4: Resumen y diagnóstico a nivel correlacional de las variables: Enseñanza aprendizaje del diseño arquitectónico en los niveles intermedios y la Educación para el Desarrollo Sostenible en las universidades de Huancayo.

\begin{tabular}{|c|c|c|c|}
\hline Variables & $\begin{array}{l}\text { Universidades } \\
\text { con facultades } \\
\text { de arquitectura }\end{array}$ & $\begin{array}{c}\text { Correlación } \\
\qquad\left(r_{s}\right)\end{array}$ & Diagnóstico \\
\hline Enseñanza & $\begin{array}{c}\text { UNCP } \\
\text { Nivel de diseño } \\
\text { intermedio }\end{array}$ & 0,14 & $\begin{array}{c}\text { Correlación } \\
\text { mínima }\end{array}$ \\
\hline $\begin{array}{l}\text { aprendizaje del diseño } \\
\text { arquitectónico en los } \\
\text { niveles intermedios y las }\end{array}$ & $\begin{array}{c}\text { UPLA } \\
\text { Nivel de diseño } \\
\text { intermedio }\end{array}$ & $-0,10$ & $\begin{array}{l}\text { Correlación } \\
\text { mínima } \\
\text { negativa }\end{array}$ \\
\hline teorías de la EDS. & $\begin{array}{c}\text { UC } \\
\text { Nivel de diseño } \\
\text { intermedio }\end{array}$ & 0,13 & $\begin{array}{c}\text { Correlación } \\
\text { mínima }\end{array}$ \\
\hline
\end{tabular}


Resumen de la prueba de la hipótesis 2: No hay correlación entre la enseñanza aprendizaje del diseño arquitectónico en los niveles de diseños intermedios y las teorías que fundamentan la Educación para el Desarrollo Sostenible en las universidades de Huancayo.

\begin{tabular}{|c|c|c|c|}
\hline $\begin{array}{l}\text { Hipótesis nula } \\
\mathrm{H}_{\mathrm{o}}: \mu=\mu_{0}\end{array}$ & $\begin{array}{l}\text { Hipótesis alterna } \\
\qquad \mathrm{H}_{1}: \mu \neq \mu_{0}\end{array}$ & $\begin{array}{c}\text { Nivel de } \\
\text { significancia }\end{array}$ & $\begin{array}{c}\text { Test } \\
\text { estadístico }\end{array}$ \\
\hline $\begin{array}{l}\text { No Existe correlación entre la } \\
\text { enseñanza aprendizaje del diseño } \\
\text { arquitectónico en los niveles } \\
\text { intermedios y las teorías de la } \\
\text { EDS. }\end{array}$ & $\begin{array}{l}\text { Existe correlación entre la } \\
\text { enseñanza aprendizaje del diseño } \\
\text { arquitectónico en los niveles } \\
\text { intermedios y las teorías de la } \\
\text { EDS. }\end{array}$ & $5 \%$ & $Z_{c}=1,23$ \\
\hline
\end{tabular}

la Educación para el Desarrollo Sostenible en las universidades de Huancayo", comprobándose así la segunda hipótesis específica.

\section{La enseñanza aprendizaje del diseño arquitectónico en los niveles avanzados y los pilares del aprendizaje cognitivo de la EDS en las universidades de Huancayo}

La tabla $N^{\circ} 5$ muestra que la Vxm (Enseñanza aprendizaje del diseño arquitectónico en los niveles avanzados) y para la Vym (Pilares del aprendizaje cognitivo de la EDS) puntuaciones para las medianas de la UNCP Me=0,16, UPLA Me=0,155, y UC concordó la mediana de $M e=0,14$; permitiendo precisar las incertidumbres entre las variables y la determinación de acuerdos bajos entre ellas en los niveles avanzados de las facultades de arquitectura investigadas.

Mientras en la tabla $N^{\circ}$ 6, las variables analizadas determinaron coeficientes de correlaciones de rho de Spearman, para los niveles avanzados de las facultades de arquitectura que concretizaron una $r S=$ 0,21 para la $U N C P, U P L A r S=-0,20$ y $\cup C r S=0,13$, que también sugirió coeficientes de correlaciones mínimas, con dirección negativas para la UNCP y UPLA, lo que detallo relaciones inversamente proporcionales y señalo la casi nula influencia que posee los pilares del aprendizaje cognitivo de la EDS en los planes curriculares, produciendo mayores deficiencias en la enseñanza aprendizaje del diseño arquitectónico en los niveles avanzados, que no materializo proyectos arquitectónicos, urbanos y de planeamiento sostenibles.

Como $1,30<2,11$, entonces el estadístico calculado cae en la zona de rechazo, por lo tanto se rechaza la alterna $\mathrm{H}_{1}$ y se acepta la hipótesis nula $\mathrm{H}_{0}$, con lo que se concluye que "No existe correlación entre la enseñanza aprendizaje del diseño arquitectónico en los niveles avanzados y los pilares del aprendizaje cognitivo de la Educación para el Desarrollo Sostenible en las universidades de Huancayo", comprobándose así la tercera hipótesis específica.

La enseñanza aprendizaje del diseño arquitectónico en los niveles profesionales y los enfoques pedagógicos de la EDS en las universidades de Huancayo

Se comprobó en los datos de la tabla $N^{\circ} 7$, valores para la UNCP Me=0,115, UPLA Me=0,165 y UC Me $=0,16$; estos eventos sugieren claramente acuerdos bajos entre las variables y la existencia de incertidumbres criticas de correspondencias en los niveles de estudios profesionales de las facultades de

Tabla № 5: Resumen y diagnóstico a nivel de la mediana de las variables: Enseñanza aprendizaje del diseño arquitectónico en los niveles avanzados y los pilares del aprendizaje cognitivo de la EDS.

\begin{tabular}{|c|c|c|c|}
\hline Variables & $\begin{array}{l}\text { Universidades } \\
\text { con facultades } \\
\text { de arquitectura } \\
\end{array}$ & $\begin{array}{c}\text { Correlación } \\
\qquad\left(r_{s}\right)\end{array}$ & Diagnóstico \\
\hline Enseñanza aprendizaje del & $\begin{array}{c}\text { UNCP } \\
\text { Nivel de diseño } \\
\text { intermedio }\end{array}$ & 0,16 & Acuerdo bajo \\
\hline $\begin{array}{l}\text { diseño arquitectónico en } \\
\text { los niveles avanzados y } \\
\text { los pilares del aprendizaje }\end{array}$ & $\begin{array}{c}\text { UPLA } \\
\text { Nivel de diseño } \\
\text { intermedio }\end{array}$ & 0,155 & Acuerdo bajo \\
\hline cognitivo de la EDS. & $\begin{array}{c}\text { UC } \\
\text { Nivel de diseño } \\
\text { intermedio }\end{array}$ & 0,14 & Acuerdo bajo \\
\hline
\end{tabular}


Tabla № 6: Resumen y diagnóstico a nivel correlacional de las variables: Enseñanza aprendizaje del diseño arquitectónico en los niveles de diseños avanzados y los Modelos del aprendizaje cognitivo de la EDS.

\begin{tabular}{|c|c|c|c|}
\hline Variables & $\begin{array}{l}\text { Universidades } \\
\text { con facultades } \\
\text { de arquitectura }\end{array}$ & $\begin{array}{c}\text { Correlación } \\
\left(r_{s}\right)\end{array}$ & Diagnóstico \\
\hline \multirow{3}{*}{$\begin{array}{l}\text { Enseñanza aprendizaje del } \\
\text { diseño arquitectónico en } \\
\text { los niveles avanzados y } \\
\text { los pilares del aprendizaje } \\
\text { cognitivo de la EDS. }\end{array}$} & $\begin{array}{c}\text { UNCP } \\
\text { Nivel de diseño } \\
\text { intermedio }\end{array}$ & $-0,21$ & $\begin{array}{l}\text { Correlación } \\
\text { mínima } \\
\text { negativa }\end{array}$ \\
\hline & $\begin{array}{c}\text { UPLA } \\
\text { Nivel de diseño } \\
\text { intermedio }\end{array}$ & $-0,20$ & $\begin{array}{l}\text { Correlación } \\
\text { mínima } \\
\text { negativa }\end{array}$ \\
\hline & $\begin{array}{c}\text { UC } \\
\text { Nivel de diseño } \\
\text { intermedio }\end{array}$ & 0,13 & $\begin{array}{l}\text { Correlación } \\
\text { mínima }\end{array}$ \\
\hline
\end{tabular}

Resumen de la prueba de la hipótesis 3: No existe correlación entre la enseñanza aprendizaje del diseño arquitectónico en los niveles avanzados y los pilares del aprendizaje cognitivo de la Educación para el Desarrollo Sostenible en las universidades de Huancayo

\begin{tabular}{|c|c|c|c|}
\hline $\begin{array}{c}\text { Hipótesis nula } \\
\mathrm{H}_{\mathrm{o}}: \mu=\mu_{0}\end{array}$ & $\begin{array}{l}\text { Hipótesis alterna } \\
\mathrm{H}_{1}: \mu \neq \mu_{0}\end{array}$ & $\begin{array}{c}\text { Nivel de } \\
\text { significancia }\end{array}$ & $\begin{array}{c}\text { Test } \\
\text { estadístico }\end{array}$ \\
\hline $\begin{array}{l}\text { No Existe correlación entre la } \\
\text { enseñanza aprendizaje del diseño } \\
\text { arquitectónico en los niveles } \\
\text { avanzados y los pilares del } \\
\text { aprendizaje cognitivo de la EDS. }\end{array}$ & $\begin{array}{l}\text { Existe correlación entre la } \\
\text { enseñanza aprendizaje del diseño } \\
\text { arquitectónico en los niveles } \\
\text { avanzados y los pilares del } \\
\text { aprendizaje cognitivo de la EDS. }\end{array}$ & $5 \%$ & $Z_{c}=1,30$ \\
\hline
\end{tabular}

Tabla № 7: Resumen y diagnóstico a nivel de la mediana de las variables: Enseñanza aprendizaje del diseño arquitectónico en los niveles profesionales y los enfoques pedagógicos de la EDS.

\begin{tabular}{|c|c|c|c|}
\hline Variables & $\begin{array}{l}\text { Universidades } \\
\text { con facultades } \\
\text { de arquitectura }\end{array}$ & $\begin{array}{c}\text { Correlación } \\
\left(r_{s}\right)\end{array}$ & Diagnóstico \\
\hline Enseñanza aprendizaje del & $\begin{array}{c}\text { UNCP } \\
\text { Nivel de diseño } \\
\text { intermedio }\end{array}$ & 0,155 & Acuerdo bajo \\
\hline $\begin{array}{l}\text { diseño arquitectónico en los } \\
\text { niveles profesionales y los } \\
\text { enfoques pedagógicos de }\end{array}$ & $\begin{array}{l}\text { UPLA } \\
\text { Nivel de diseño } \\
\text { intermedio }\end{array}$ & 0,165 & Acuerdo bajo \\
\hline la EDS. & $\begin{array}{c}\text { UC } \\
\text { Nivel de diseño } \\
\text { intermedio }\end{array}$ & 0,16 & Acuerdo bajo \\
\hline
\end{tabular}

arquitectura analizados.

En la tabla $N^{\circ} 8$, los coeficientes de rho de Spearman determinaron para las variables analizadas en los niveles profesionales de las facultades de arquitectura, correlaciones de $r S=-0,18$ para la UNCP, UPLA $r S=$ 0,16 y $\cup C r S=0,07$, con la tendencia a confirmar mínimas correlaciones y con direcciones negativas o inversamente proporcionales, especialmente para los casos de la UNCP y UPLA, que detectaron las menores implicancia que tuvieron los enfoques pedagógicos de la Educación para el Desarrollo Sostenible, que concretizo la ausencia de un buen nivel académico en la enseñanza del diseño arquitectónico en los niveles profesionales y por ende la concretización de proyectos urbano rurales no innovadores de carácter internacional, por la carencia de los enfoques sostenibles.

Como $1,01<1,39$, entonces el estadístico calculado 
Tabla N $N^{\circ}$ 8: Resumen y diagnóstico a nivel correlacional de las variables: Enseñanza aprendizaje de la arquitectura en los niveles profesionales y los enfoques pedagógicos de la EDS.

\begin{tabular}{|c|c|c|c|}
\hline Variables & $\begin{array}{l}\text { Universidades } \\
\text { con facultades } \\
\text { de arquitectura }\end{array}$ & $\begin{array}{l}\text { Correlación } \\
\qquad\left(r_{s}\right)\end{array}$ & Diagnóstico \\
\hline Enseñanza aprendizaje del & $\begin{array}{l}\text { UNCP } \\
\text { Nivel de diseño } \\
\text { intermedio }\end{array}$ & $-0,18$ & $\begin{array}{l}\text { Correlación } \\
\text { mínima } \\
\text { negativa }\end{array}$ \\
\hline $\begin{array}{l}\text { diseño arquitectónico en los } \\
\text { niveles profesionales y los } \\
\text { enfoques pedagógicos de }\end{array}$ & $\begin{array}{l}\text { UPLA } \\
\text { Nivel de diseño } \\
\text { intermedio }\end{array}$ & $-0,16$ & $\begin{array}{l}\text { Correlación } \\
\text { mínima } \\
\text { negativa }\end{array}$ \\
\hline la EDS. & $\begin{array}{l}\text { UC } \\
\text { Nivel de diseño } \\
\text { intermedio }\end{array}$ & 0,07 & $\begin{array}{c}\text { Correlación } \\
\text { mínima }\end{array}$ \\
\hline
\end{tabular}

Resumen de la prueba de la hipótesis 4: No hay correlación entre la enseñanza aprendizaje del diseño arquitectónico en los niveles profesionales y los enfoques pedagógicos de la Educación para el Desarrollo Sostenible en las universidades de Huancayo.

\begin{tabular}{|c|c|c|c|}
\hline $\begin{array}{c}\text { Hipótesis nula } \\
\mathrm{H}_{0}: \mu=\mu_{0}\end{array}$ & $\begin{array}{l}\text { Hipótesis alterna } \\
\qquad \mathrm{H}_{1}: \mu \neq \mu_{0}\end{array}$ & $\begin{array}{c}\text { Nivel de } \\
\text { significancia }\end{array}$ & $\begin{array}{c}\text { Test } \\
\text { estadístico }\end{array}$ \\
\hline $\begin{array}{l}\text { No Existe correlación entre la } \\
\text { enseñanza aprendizaje del diseño } \\
\text { arquitectónico en los niveles } \\
\text { profesionales y los enfoques } \\
\text { pedagógicos de la EDS. }\end{array}$ & $\begin{array}{l}\text { Existe correlación entre la } \\
\text { enseñanza aprendizaje del diseño } \\
\text { arquitectónico en los niveles } \\
\text { profesionales y los enfoques } \\
\text { pedagógicos de la EDS. }\end{array}$ & $5 \%$ & $Z_{c}=1,01$ \\
\hline
\end{tabular}

cae en la zona de rechazo, por lo tanto se rechaza la alterna $\mathrm{H}_{1}$ y se acepta la hipótesis nula $\mathrm{H}_{0}$, con lo que se concluye que "No hay correlación entre la enseñanza aprendizaje del diseño arquitectónico en los niveles profesionales y los enfoques pedagógicos de la Educación para el Desarrollo Sostenible en las universidades de Huancayo", comprobándose así la cuarta y última hipótesis específica.

\section{DISCUSIÓN}

Para las variables, Enseñanza aprendizaje en los talleres de diseño arquitectónico del nivel básico I, II y III y la Fundamentación epistemológica de la EDS de las facultades de arquitectura inferidas, se encontraron un no entendimiento entre las variables y la confirmación de acuerdos bajos entre ellas, inclusive de una de carácter crítico, (tabla $N^{\circ} 1$ ) asimismo, se dio las tendencias a coeficientes de correlaciones con valoraciones muy mínimas y con direcciones dominantemente positivas (tabla $\mathrm{N}^{\circ} 2$ ).

Estos resultados detallaron que los diseños curriculares y los sílabos en referencia a la enseñanza aprendizaje de la arquitectura en los talleres de diseños de nivel básico, se concretizaron al margen de las fundamentaciones epistemológicas que se encuentran en el marco de la EDS. La planificación de la enseñanza aprendizaje en los talleres de diseño plantean propuestas compositivas de peculiaridades bidimensionales, tridimensionales y espaciales, dejando de lado, materiales como son los desechos urbanos, cuyo costo es ínfimo y que tan solo se tienen que operar un proceso de reciclado o reutilización para construir composiciones creativas e innovadoras, y que no se aprecia en la enseñanza por los docentes. De igual manera, se muestran concretizaciones de proyectos arquitectónicos básicos que mínimamente toman en cuenta a la radiación solar, la presión atmosférica, la temperatura y las precipitaciones pluviales en sus propuestas proyectuales, situación que se debe principalmente al escaso conocimiento que tienen los docentes sobre esta temática ambiental.

Las propuestas arquitectónicas se realizan haciendo el no uso racional del agua, no realizan tratamientos de aguas residuales, no hay ahorro de energía o la notoria ausencia de estudios de impactos ambientales y de calidad ambiental; la concreción de tipologías arquitectónicas eco eficientes, son temáticas ausentes del proceso de enseñanza, debido a que los docentes no cuentan con la especialización respectiva. Esto quiere decir, que el diseño curricular y los sílabos y el mismo proceso de enseñanza no toma en cuenta, por ejemplo, lo que expresa la Agenda 21 (8) o el Informe Bruntlad o Nuestro Futuro Común (9). 
Estos hallazgos se pueden considerar como contradictorios con casi todos los enfoques teóricos que tratan la educación desde el paradigma de desarrollo sostenible, como hacen referencia Ciurana y Motta (10) y otras fuentes. De igual manera, Osalde (11) se aúna en afirmar los desgastados y cuestionados métodos de enseñanza que se imparten aún en las cátedras de diseño arquitectónico, están permitiendo avizorar un futuro medio ambiental muy incierto para la región Junín y el planeta.

Como se analizó anteriormente, se afirma la hipótesis planteada que: "la planificación de la enseñanza aprendizaje de la arquitectura en los talleres de diseño arquitectónico de los niveles básicos se mantienen una posición marginal de la significancia que poseen las teorías educativas y pedagógicas que han concebido el nuevo paradigma educativo del Desarrollo Sostenible y esta situación se ve traducida propuestas bidimensionales, tridimensionales y espaciales carentes del paradigma sostenible y en la deficiente y no actualizada enseñanza de los diseños, como también en los procesos de gestión académica del diseño arquitectónico".

Por otro lado, para las variables: Enseñanza aprendizaje en los talleres de diseño arquitectónico del nivel intermedio IV, $\mathrm{VI}$ y $\mathrm{VI}$ y de la arquitectura y las Teorías que fundamentan la EDS, permiten afirmar que existen manifiestas tendencias a acuerdos bajos entre las variables, (tabla $N^{\circ} 3$ ) además existe un coeficiente de correlación también muy mínima con dirección hacia lo positivo predominantemente (tabla $\mathrm{N}^{\circ} 4$ ).

Los datos confirmaron que los diseños curriculares y los sílabos en concordancia a la enseñanza aprendizaje de la arquitectura en los talleres de diseño que pertenecen al nivel intermedio, se realizan en cierto modo aislados de las teorías y enfoques pedagógicos de la sustentabilidad, las cuales se encuentran en el marco de la Educación para el Desarrollo Sostenible; es decir, no existen los esfuerzos de poder hacer de la enseñanza del diseño instrumentos de cambio y de desarrollo sostenible. Sauvé (12) por ese motivo se percibió las mínimas relaciones que existen entre las variables de estudio, que encuentra su fundamento en las propuestas de tipologías arquitectónicas sin criterios de innovación y creatividad, incluso, los documentos educativos como el diseño curricular, sílabos y otros adolecen de estos criterios. De igual manera, el resultado hace ver las limitaciones pedagógicas que posee el recurso humano; que visto desde la óptica de la enseñanza de los talleres de diseño, estos aún permanecen al margen de los nuevos enfoques de desarrollo sostenible que tiene la educación superior en arquitectura en estos momentos Mosquera (13).

También se comprobó la ausencia propuestas de tipologías arquitectónicas que tomen en cuenta las energías renovables como las energías solar, eólica, la hidro energía y la biomasa como fuente energética y sobre todo que la ciudad se inserte a la naturaleza o viceversa de una manera sostenible. Con características similares se presenta en otros talleres de diseño, donde el uso de materiales constructivos sostenibles, como la madera, la tierra o barro, el bambú y los materiales reciclados y el conjunto de tecnologías del que están conformados estos, no son tomados en cuenta en la formación de los estudiantes, por el desconocimiento de la naturaleza, mecánica y tecnologías de los materiales mencionados por parte de los docentes.

Se observo que el uso de la energía solar térmica y termoeléctrica, la energía fotovoltaica y la energía eólica solar no se plasman en las propuestas de las tipológicas arquitectónicas de este nivel y en la formación de los estudiantes, por la carencia de docentes especializados en estas tecnologías, lo que denota la ausencia de los enfoques pedagógicos sistémico y holístico que requiere la arquitectura actual, Mosquera (13) como base para abordar un mundo global, y para aplicar el pensamiento complejo, Morín (14) tan importante en la enseñanza de los diseños arquitectónicos actuales.

La declaración de estos argumentos permiten responder a la hipótesis y plantear que: "la planificación de la enseñanza aprendizaje de la arquitectura en los talleres de diseño de los niveles intermedios IV, V y VI, también se encuentra con una tendencia a mantenerse aislados de la significancia de las nuevas teorías educativas y del paradigma del desarrollo sostenible, hecho que se contrasto en las propuestas de las tipologías arquitectónicas y en el diseño curricular, sílabos y otros documentos de gestión educativa y pedagógica".

Infiriendo las variables: Enseñanza aprendizaje en los talleres de diseño arquitectónico del nivel avanzado VII, VIII y IX y los Pilares del aprendizaje cognitivo de la Educación para el Desarrollo Sostenible, se confirmó acuerdos bajos y separaciones alta de datos al interior de cada variable como entre ellas, (ver tabla 5) además existe un coeficiente de correlación mínima con una clara dirección negativa (tabla $\mathrm{N}^{\circ} 6$ ).

Estos datos mostraron que los diseños curriculares y los sílabos en referencia a la enseñanza aprendizaje de la arquitectura en los talleres de diseño que corresponden al nivel avanzado, se desarrollan sin el discurso académico de tomar en cuenta los pilares del aprendizaje cognitivo; es el caso, de una educación de aprender a conocer, aprender a hacer, aprender a vivir juntos, aprender a ser y aprender a transformarse a uno mismo y la sociedad Delors (2). La ausencia de estos conceptos se observa en el currículo de estudios y todo el conjunto de documentos que lo conforman en referencia a la enseñanza aprendizaje de la 
arquitectura y por ende del diseño arquitectónico.

En este sentido, en los talleres se enseñan a diseñar tipologías arquitectónicas de peculiaridades financieras, deportivas, turísticas, industriales y arquitectura cívica, manteniendo los mismos enfoques y estándares pedagógicos que han perdurado por décadas, dejando de lado, la arquitectura sostenible, con las mismas peculiaridades en las propuestas en la gestión y planeamiento de sistemas urbanos rurales en los espacios distritales, provinciales y regionales, se conciben alejados del discurso y prácticas del eco urbanismo y del planeamiento sostenible y de gestión del patrimonio cultural, acciones que se deben a falta de especialización de estos nuevos enfoques y las serias carencias de los profesores que imparten estos conocimientos, eventos que se confirman en los proyectos urbanos y del planeamiento propuestos y que además se confirma en el diseño curricular y en los sílabos de las asignaturas respectivas; este argumento se apoya en el concepto: que no existe un esfuerzo de poder hacer de la enseñanza del diseño arquitectónico un instrumento de cambio y de desarrollo sostenible Sauvé (12).

Por ese motivo se observó las relaciones mínimas que existe entre la variables de estudio, igualmente, el resultado hace ver las limitaciones académicas que posee el recurso humano, que desde la óptica de la enseñanza de la arquitectura permanece al margen de los nuevos enfoques de desarrollo que posee la educación superior en estos momentos Mosquera (13).

Respecto a la hipótesis planteada se hace pertinente responder con los mismos argumentos que: "la planificación de la enseñanza aprendizaje de la arquitectura en los talleres de diseño de los niveles VII, VIII y IX se encuentran en cierta forma al margen de la significancia de los pilares el aprendizaje cognitivos que requiere la nueva educación". Los diseños y planes curriculares, los sílabos y otros documentos de carácter de gestión educativa carecen de los argumentos anotados, cuya replica también se comprueba en la enseñanza aprendizaje de la arquitectura y del diseño arquitectónico.

Finalmente, entre las variables: Enseñanza aprendizaje del diseño arquitectónico en los niveles profesionales y los enfoques pedagógicos de la Educación para el Desarrollos Sostenible, se sugieren acuerdo bajos, (ver tabla 7) además se determinó que existe un coeficiente de correlaciones mínimos. (ver tabla 8)

En estos niveles profesionales de diseño arquitectónico, la enseñanza de los proyectos de sistemas urbanos, rurales y urbanos rurales y que se contextualiza en el ámbito internacional, dejan de lado corrientes como la naturalista, la conservacionista / recursista, la resolución de problemas, sistémica, la holística, entre otros, no sabiendo la importancia que poseen estas en la pedagogía del diseño arquitectónico nivel de estudiantes casi egresados, y que busca un perfil profesional que se enraícen de manera coherente en el espacio de trabajo y en el respeto al medio ambiente Sauve (12).

Los resultados mostraron que los diseños curriculares y los sílabos en referencia a la enseñanza aprendizaje del diseño arquitectónico en estos niveles se realizan también con la casi carencia de las corrientes pedagógicas actuales mencionadas en el ítem anterior y que se encuentran en el marco de la Educación para el Desarrollo Sostenible, aun sabiendo que esta se constituye en un objetivo en sí misma, debido a que posee un campo conceptual propio y una capacidad de transformación de las condiciones materiales destinadas a un ambiente menos contaminado y depredado Gutiérrez y Pozo (15). De igual manera, esta no se encuentra ni contextualizada en los documentos de gestión educativa, que requiere y urge una enseñanza realizada con fundamentos científicos.

De igual manera, las secuelas hacen ver las posibilidades de capacidad que posee el recurso humano de los docentes, que desde la óptica de la enseñanza, permanecen también al margen de los nuevos enfoques de desarrollo de la educación superior Mosquera (13).

Desde la óptica analizada se contextualiza una respuesta a la hipótesis y se plantea que: "la planificación de la enseñanza aprendizaje de la arquitectura y por ende del diseño arquitectónico en los niveles profesionales también se encuentra aislado de los enfoques pedagógicos como es la naturalista, la corriente sistémica, la científica, la holística entre otros, cuya significancia en la documentación de gestión educativa y pedagógica del taller de diseño profesional se hace imprescindible y del cual se carece".

\section{REFERENCIAS BIBLIOGRÁFICAS}

1. Organización de las Naciones Unidas para la Educación, la Ciencia y la Cultura [Internet]. Santiago de Chile: UNESCO; [Citado el 20 de diciembre de 2009]. Década de la Educación para el Desarrollo Sostenible: 2005-2014 [1 pantalla]. Disponible en: http://www.unesco. org/new/es/santiago/education/education-forsustainable-development/decade-of-educationfor-sustainable-development-desd/

2. United Nations Educational, Scientific and Cultural Organization [Internet]. Paris: UNESCO; [Citado el 20 de diciembre de 2009]. Education for 
Sustainable Development (ESD). Disponible en: www.unesco.org/education/desd. 3. Palmer J. Environmental Education in the 21 st Century. Theory, Practice, Progress \& Promise. Londres: Routledge; 1998.

4. Villegas L, Marroquín R, Del Castillo V, Sánchez R. Teoría y praxis de la investigación científica. Lima: San Marcos; 2011.

5. Carrasco S. Metodología de la investigación científica. Lima: San Marcos; 2006.

6. Pino R. Metodología de la Investigación. Lima: San Marcos; 2008.

7. Hernández R, Fernández C, Baptista P. Metodología de la Investigación. 5a ed. México: McGraw-Hill; 2010.

8. Naciones Unidas [Internet]. New York: Departamento de asuntos económicos y sociales; [Citado el 20 de noviembre de 2005]. Agenda 21 [1 pantalla]. Disponible en: http://www.un.org/ spanish/esa/sustdev/agenda21/

9. World Commission on Environment and Development. Our Common Future. Washington D.C. WCED/Oxford University Press; 1987.

10. Morin E, Ciurana ER, Motta RD. Educar en la era planetaria: El pensamiento complejo como un método de aprendizaje en el error y la incertidumbre humana. $\rceil^{\mathrm{a}}$ ed. Valladolid: Universidad de Valladolid; 2003.

11. Osalde A. Nuevas formas de enseñar aprender el Diseño Arquitectónico en el Taller de Composición. México D.F.; 2009.

12. Sauvé L. Uma cartografía das correntes em educação ambiental. En: Sato $M$, Carvalho I. Educação ambiental, Pesquisa e desafios, São Paulo: Artmed; 2005.

13. Mosquera J. Epistemología y didáctica del proceso de enseñanza-aprendizaje en arquitectura y diseño industrial. Revista Científica Guillermo de Ockham. 2009; 7(2): 33-49.

14. Morín E. Introducción al pensamiento complejo. 9a ed. Barcelona: Antropos; 2003.

15. Pozo T, Benayas J, Gutiérrez J. Modelos de calidad y prácticas evaluativas predominantes en los equipamientos de educación ambiental. Tópicos en educación ambiental. 2011 ; 1(2): 49-64. 